\title{
de Bruijn notation as a nested datatype
}

\author{
Richard S. Bird \\ Programming Research Group, Oxford University \\ Wolfson Building, Parks Road, Oxford, OX1 3QD, UK \\ Ross Paterson \\ Department of Computer Science, City University, \\ Northhampton Square, London EC1V OHB, UK
}

"I have no data yet. It is a capital mistake to theorise before one has data."

Sir Arthur Conan Doyle

The Adventures of Sherlock Holmes

\begin{abstract}
de Bruijn notation is a coding of lambda terms in which each occurrence of a bound variable $x$ is replaced by a natural number, indicating the 'distance' from the occurrence to the abstraction that introduced $x$. One might suppose that in any datatype for representing de Bruijn terms, the distance restriction on numbers would have to maintained as an explicit datatype invariant. However, by using a nested (or non-regular) datatype, we can define a representation in which all terms are well-formed, so that the invariant is enforced automatically by the type system.

Programming with nested types is only a little more difficult than programming with regular types, provided we stick to well-established structuring techniques. These involve expressing inductively defined functions in terms of an appropriate fold function for the type, and using fusion laws to establish their properties. In particular, the definition of lambda abstraction and beta reduction is particularly simple, and the proof of their associated properties is entirely mechanical.
\end{abstract}

\section{Introduction}

A standard representation of lambda terms, with variables of type $v$, in Haskell involves essentially the following datatype:

$$
\text { data Term } v=\operatorname{Var} v|\operatorname{App}(\operatorname{Term} v, \operatorname{Term} v)| \operatorname{Lam} v(\operatorname{Term} v)
$$

The problem with the standard representation is that while abstraction is easy to implement, application is not. Application of a lambda term Lam $x b$ to an argument $t$ involves substituting $t$ for all free occurrences of $x$ in $b$. Care has to be taken to avoid the capture of free variables in $t$ by bound variables in $b$. To overcome this problem, N. de Bruijn (de Bruijn, 1972) proposed a notation for lambda expressions in which bound variables do not occur. In his notation, no variable appears after 
the constructor Lam, and bound variables appear as natural numbers. The number assigned to an occurrence of a bound variable $x$ is the depth of nesting of Lam terms between that occurrence and the (closest) binding occurrence of $x$. For example,

$$
\lambda x . x(\lambda y . x y(\lambda z . x y z))
$$

translates to

$$
\lambda .0(\lambda .10(\lambda .210))
$$

This example is taken from (Paulson, 1996), which discusses de Bruijn notation in detail.

If one wants to represent lambda terms involving both bound and free variables in the de Bruijn style, then the declaration of Term $v$ has to be changed. One possibility, used in (Paulson, 1996), is to have two kinds of variable: free variables drawn from $v$, and bound variables drawn from Int. Another possibility is to use a datatype declaration

$$
\begin{aligned}
& \text { data Term } v=\operatorname{Var} v \mid \text { App }(\text { Term } v, \text { Term } v) \mid \operatorname{Lam}(\text { Term }(\operatorname{Incr} v)) \\
& \text { data Incr } v=\text { Zero } \mid \text { Succ } v
\end{aligned}
$$

In the body of a lambda abstraction, the set of variables is augmented with an extra element, the variable bound by the lambda. This variable is denoted by Zero; each free variable $x$ is renamed Succ $x$ inside the lambda. For example, the terms $\lambda x . x$ and $\lambda x . \lambda y . x$ are represented as

$$
\operatorname{Lam}(\operatorname{Var} \text { Zero) and Lam (Lam (Var (Succ Zero))) }
$$

The term $\lambda x . \lambda y . x y z$, containing a free variable $z$, may be represented as the following element of Term Char:

$$
\operatorname{Lam}\left(\operatorname{Lam}\left(\operatorname{App}\left(\text { App (Var (Succ Zero), Var Zero), Var }\left(\operatorname{Succ}\left(\operatorname{Succ}{ }^{\prime} z^{\prime}\right)\right)\right)\right)\right)
$$

The type Term is an example of a nested datatype (Bird \& Meertens, 1998) because its definition has a recursive use with a different argument from the left-hand side. Such definitions are also sometimes called non-regular.

Our aim in this paper is to study this novel representation of lambda terms, and to give the implementations of abstraction and application. Useful and interesting examples of nested datatypes have been rather thin on the ground until recently, and de Bruijn notation gives us an excellent opportunity to explore the theory in the context of a specific example. We believe that the right way to proceed into the largely uncharted territory of nested types is to stick to the structuring principles provided by the now well-established theory of regular datatypes. This theory is reviewed briefly in Section 2. In Section 3 we introduce the type of lambda terms, and set up appropriate machinery for defining functions over this type. The implementations of abstraction and application are given in Section 4. In the final section, we will generalise what we have learnt to cover an extension of de Bruijn's notation.

Another aim of the paper concerns proof. In our view, equational properties of functions are most easily proved when functions are defined as combinations of 
other functions, using functional composition rather than application as the primary combining form. As a consequence, proof by induction is replaced by appeal to general equational laws that make up standard theory. This material is also reviewed briefly in Section 2. Proofs of the various equations were generated using the simple automatic calculator described in (Bird, 1998); we include a selection of them.

All programs in typewriter font are expressed in Hugs 1.3c (Jones, 1998), an extension of Haskell that provides a more flexible typing discipline.

\section{Preliminaries}

Let us begin, not with Term, but with the simpler inductive datatype of binary trees (which is equivalent to Term without the difficult Lam case):

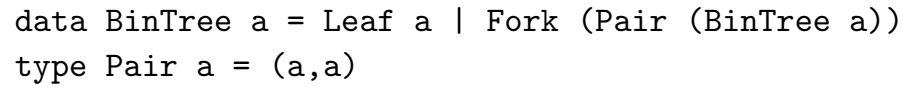

By default, Haskell allows Leaf and Fork to be non-strict functions, so the declaration above captures partial and infinite trees as well as finite ones. However, although all functions defined in this paper are legal Haskell (extended with a more general typing discipline), we are only concerned with datatypes that are flat sets, and functions that are total in the set-theoretic sense. Thus, all functions are considered to be strict, as in ML. This will enable us to state equational laws without mentioning strictness conditions explicitly.

\subsection{Functors}

For each datatype constructor*, there is a corresponding action on functions, which preserves the shape of a data structure while replacing elements within it. The classic example is the map function on lists, and functional programmers call these actions mapping functions. For the type constructor Pair, the mapping function is

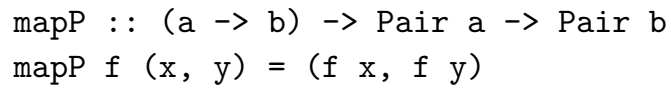

The mapping function on binary trees is:

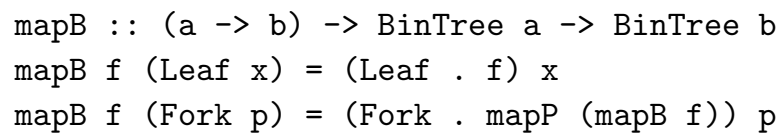

The slightly unusual form of the right-hand sides is intended to suggest the functionlevel equations

$$
\begin{aligned}
& \text { mapB } f \cdot \text { Leaf }=\text { Leaf } \cdot f \\
& \text { mapB } f \cdot \text { Fork }=\text { Fork } \cdot \operatorname{map} P(\operatorname{map} B f)
\end{aligned}
$$

* We do not consider type constructors that include function types. 
Category theorists refer to the combination of type constructor and map function as a functor. Hence the following laws, satisfied by any mapping function, are called functor laws:

$$
\begin{aligned}
\operatorname{map} B i d & =i d \\
\operatorname{map} B(f \cdot g) & =\operatorname{map} B f \cdot \operatorname{map} B g
\end{aligned}
$$

A further property, called naturality, plays an important role in many calculations. A polymorphic function $f:: M a \rightarrow N a$, where $M$ and $N$ are given type constructors, may be viewed as a collection of functions, one for each instantiation of the type variable $a$. Because $f$ is polymorphic, i.e. defined independently of $a$, these instances are related by the following naturality condition:

$$
\operatorname{map} N k \cdot f=f \cdot \operatorname{map} M k
$$

for all functions $k$, where $\operatorname{map} M$ and $\operatorname{map} N$ are the map functions for the type constructors $M$ and $N$, respectively. Such functions $f$ are called natural transformations.

As one example, any function of type

$$
\text { flatten : : BinTree a } \rightarrow[a]
$$

is a natural transformation, with naturality property

$$
\text { map } f \cdot \text { flatten }=\text { flatten } \cdot \operatorname{map} B f
$$

Similarly, the naturality of the BinTree constructors Leaf $:: a \rightarrow$ BinTree $a$ and Fork :: Pair (BinTree $a) \rightarrow$ BinTree $a$ is expressed by equations (1) and (2), which define the action mapB. Note that the action on functions corresponding to the identity type constructor is the identity, and a composition of type constructors corresponds to a composition of actions.

\subsection{Folds}

The second general operator generalises the foldr function on lists. For binary trees, the operator is

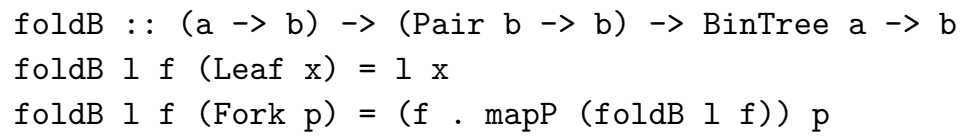

The fold operator takes a function argument for each constructor of the datatype. Its action to replace the constructors in its input with the corresponding functions. Often the effect is to reduce the data structure to a summary value, as in the first two of the following examples:

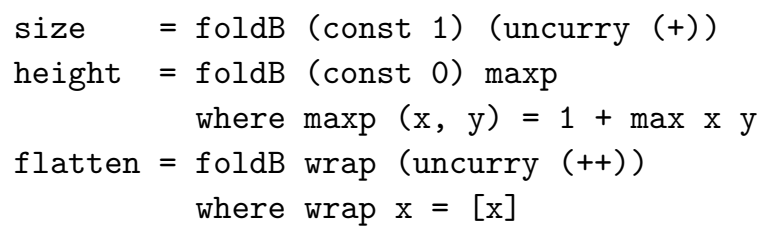


A fundamental property of all fold operators is that they produce the unique function satisfying the above defining equations. From this follows a trio of useful calculational laws. The simplest is the identity law, which for binary trees is

$$
\text { foldB Leaf Fork }=i d
$$

The other two laws are more powerful, and heavily used in calculations. The fusion law states that

$$
h \cdot \text { foldB } l f=\text { foldB } l^{\prime} f^{\prime} \Leftarrow\left\{\begin{array}{l}
h \cdot l=l^{\prime} \\
h \cdot f=f^{\prime} \cdot \operatorname{map} P h
\end{array}\right.
$$

The map-fusion law states that

$$
\text { foldB } l f \cdot \operatorname{map} B h=\operatorname{foldB}(l \cdot h) f
$$

An immediate consequence of map-fusion and the identity law is an alternative definition of $\operatorname{map} B$ as a fold:

$$
\operatorname{map} B h=\operatorname{foldB}(\text { Leaf } \cdot h) \text { Fork }
$$

The map operator for each regular datatype may be defined as fold in this way, but this does not hold for nested datatypes.

Fusion laws, functor properties, and naturality conditions, are all we need for a powerful generic equational theory of inductive datatypes. For further details, see (Bird \& de Moor, 1997).

\subsection{Monads}

Monad operations provide a useful way of structuring many programs. Functional programmers are introduced to monads as a type constructor with a certain binding operation. Category theorists use a function-level definition, which is also more convenient for calculations. A monad is defined as a type constructor $M$ with a mapping function mapM and two operations

$$
\begin{array}{lll}
\text { unit } & :: & a \rightarrow M a \\
\text { join } & :: & M(M a) \rightarrow M a
\end{array}
$$

These natural transformations are required to satisfy the following coherence laws:

$$
\begin{aligned}
\text { join } \cdot \text { mapM unit } & =i d \\
\text { join } \cdot \text { unit } & =i d \\
\text { join } \cdot \text { mapM join } & =\text { join } \cdot \text { join }
\end{aligned}
$$

In total, there are seven laws available for reasoning about a monad: the three coherence laws, the two naturality laws for unit and join, and the two functor laws for $\operatorname{map} M$, the mapping function associated with $M$.

A standard example of a monad is the list type constructor, with unit returning a singleton list, and concat as the join operation. Binary trees also form a monad, with unit Leaf and the following join function: 


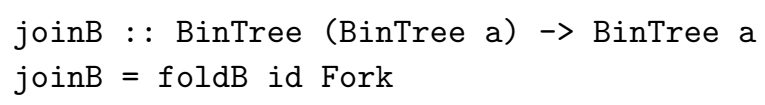

As we will see, lambda terms also form a monad; the unit and join operations on lambda terms will be needed in the definition of lambda abstraction and application. See (Bird, 1998) for further discussion of monads and monad laws, and the different ways one can describe them.

\section{3 de Bruijn notation}

We can follow the same steps with the type Term $a$ of lambda terms over a type $a$ :

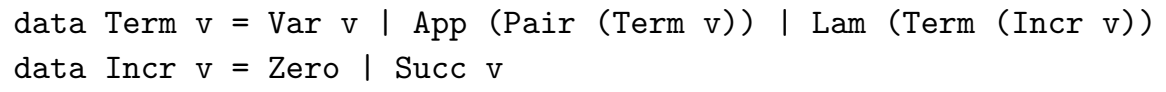

\subsection{Maps}

The first step is to identify the map operators for the newly introduced types. The mapping function corresponding to Incr is straightforward:

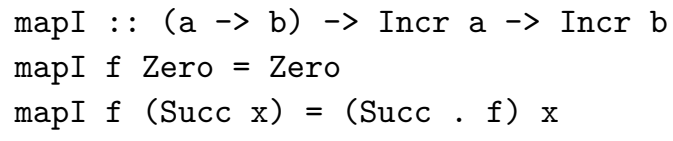

As we might expect, Term is more interesting:

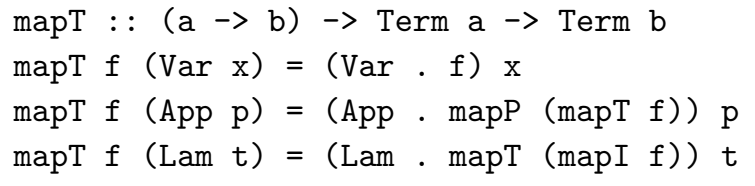

Note the change of argument of mapT in the Lam case: the required mapping function for Term (Incr a) is mapT (mapIf). As a result, mapT leaves bound variables unchanged, and replaces only free variables. In the nested definition, bound variables have become part of the shape of a term.

Note also that the argument of mapT in the Lam case also has a different type, namely Incr $a \rightarrow$ Incr $b$, but this is an instance of the declared signature. The definition of map $T$ makes use of polymorphic recursion; it is the first function in this paper whose type signature cannot be omitted.

\subsection{Folds}

The definition of the fold function for Term follows from the principle of replacing constructors by functions:

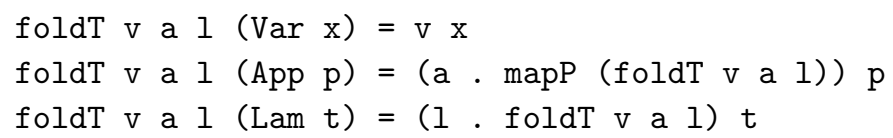


Unfortunately, the last line of this definition will not pass a standard Haskell typechecker: if foldT $v$ a $l$ is applied to a term of type Term $V$ for some type $V$, then the foldT $v$ a $l$ on the right side is applied to a term of type Term (Incr V). Hence the argument functions $v, a$ and $l$ must be applicable at a range of different types; effectively, they must be polymorphic. Haskell's language of types cannot express this without an extension called rank-2 type signatures (McCracken, 1984). Such signatures have been implemented in GHC and also in Hugs 1.3c (Peyton Jones et al. , 1998; Jones, 1998). In the syntax of Hugs 1.3c, foldT can be made acceptable by adding the following type signature:

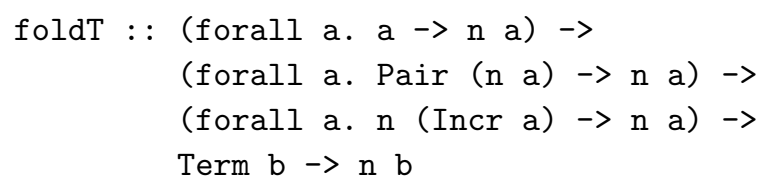

Here the variable $n$ denotes an arbitrary type constructor.

As a consequence of the arguments being natural transformations, foldT $v$ a $l$ is a natural transformation, with associated property

$$
\operatorname{map} N k \cdot \operatorname{foldT} v a l=\operatorname{foldT} \text { val } \cdot \operatorname{map} T k
$$

The naturality law of foldT does not hold for regular datatypes, such as binary trees or lists, because the argument of the fold is not required to be natural.

The above naturality condition implies that no instance of foldT can manipulate the values of free variables. As a result, we cannot define all the functions we would like on terms as instances of foldT. This phenomenon motivates a more general definition of the fold operator on nested datatypes such as Term; we will call it gfold for generalised fold:

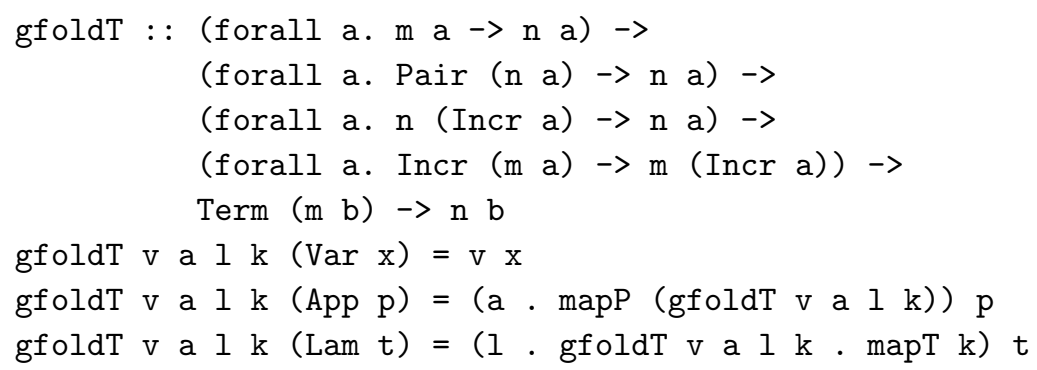

The two additional ingredients in the definition of gfoldT are, firstly, that the argument of $v$ is generalised from $a$ to $m a$ for an arbitrary type constructor $m$ and, secondly, that an extra argument $k$ is provided for the fold. To explain the role of the extra function $k$, observe that a lambda term with variables drawn from $m b$ has type

$$
\operatorname{Term}(\operatorname{Incr}(m b))
$$

Applying $\operatorname{map} T k$ to this lambda term produces an element of type

$\operatorname{Term}(m(\operatorname{Incr} b))$ 
Applying gfoldT v a $l k$ to this element produces an element of type

$$
n(\operatorname{Incr} b)
$$

This is the correct type for an argument of $l$. More details of generalised folds and their properties may be found in a companion paper (Bird \& Paterson, 1998).

The arguments to gfoldT are natural transformations, and the result is also a natural transformation. Thus, if gfoldT valk :: Term $(M b) \rightarrow N b$, we have

$$
\operatorname{map} N k \cdot g \text { foldT valk } k \text { gfoldT valk } \operatorname{map} T(\operatorname{map} M k)
$$

for all $k$, where $\operatorname{map} M$ and $\operatorname{map} N$ are the mapping functions associated with the type constructors $M$ and $N$.

The advantage of the generalised fold resides in the extra degree of freedom for selecting the type constructor $m$. In theory, we can take $m=I d$, the identity type constructor, and so obtain

$$
\text { foldTval = gfoldTvalid }
$$

as a special case. Thus gfoldT generalises foldT. Another instance of gfoldT takes both $m$ and $n$ to be constant type constructors, delivering specific types for all arguments. However, type constructor polymorphism in Haskell is limited, in that type constructor variables may only be instantiated to datatype constructors (possibly partially applied). The alternative to expressing these special cases by installing $I d$ and Const as new datatype constructors is to define specialised versions of gfoldT. For example, the following version corresponds to the constant type constructors case:

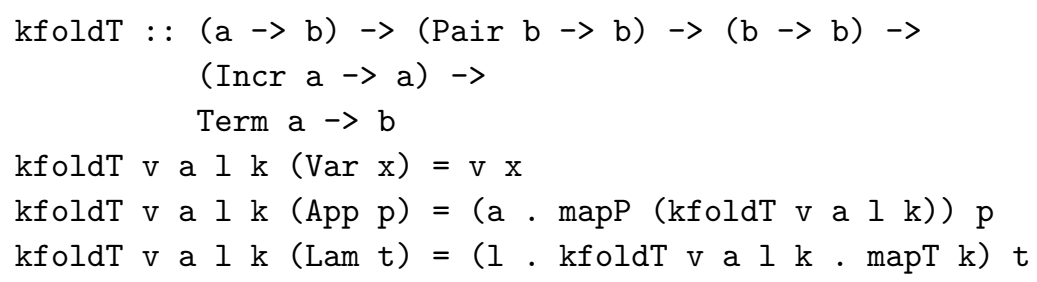

Note that $k f o l d T$ has exactly the same definition as gfoldT, but a different (more specific) type. For example, we can convert a lambda term to a string by

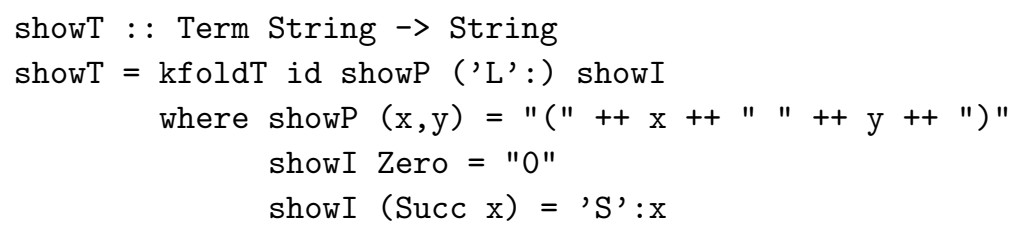

In particular, we can use showT to convert an element of type Term Char to a string in which individual character variables are printed without their quotes:

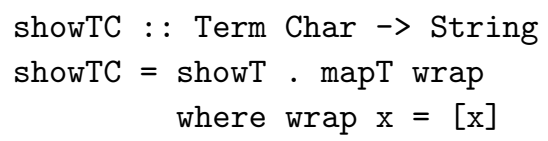


For example, applying showTC to

$$
\operatorname{Lam}\left(\operatorname{App}\left(\operatorname{Var} Z \operatorname{Zero}, \operatorname{App}\left(\operatorname{Var}\left(\operatorname{Succ}{ }^{\prime} x^{\prime}\right), \operatorname{Var}\left(\operatorname{Succ}{ }^{\prime} y '\right)\right)\right)\right)
$$

produces the string $\mathrm{L}\left(\mathrm{O}\left(\mathrm{x}^{\prime} \mathrm{y}^{\prime}\right)\right)$ ).

The function gfoldT satisfies similar fusion laws to those discussed above for binary trees. Such laws are proved from the fact that gfoldT is the unique function satisfying its defining equation. (This can be established by induction over terms.)

In particular, the identity law states that

$$
\text { gfoldT Var App Lam id }=\text { id }
$$

The map-fusion law states that

$$
\text { gfoldT valk } \operatorname{map} T h=\operatorname{gfoldT}(v \cdot h) \text { a } l k^{\prime} \Leftarrow k \cdot \operatorname{map} I h=h \cdot k^{\prime}
$$

The fold-fusion law is the following: suppose we have the typing

$$
\text { gfoldT valk :: Term }(M a) \rightarrow N a
$$

Then

$$
\begin{aligned}
h \cdot \text { gfoldT valk } & =\text { gfoldT } v^{\prime} a^{\prime} l^{\prime}\left(\operatorname{map} M k^{\prime} \cdot k\right) \\
& \Leftarrow\left\{\begin{aligned}
& h \cdot v=v^{\prime} \\
& h \cdot a= a^{\prime} \cdot \operatorname{map} P h \\
& h \cdot l= l^{\prime} \cdot h \cdot \operatorname{map} N k^{\prime}
\end{aligned}\right.
\end{aligned}
$$

The proof consists of simple calculations to show that $h \cdot g$ foldT $v$ a $l k$ satisfies the defining equations of gfoldT $v^{\prime} a^{\prime} l^{\prime}\left(\operatorname{map} M k^{\prime} \cdot k\right)$. The Lam case is the longest:

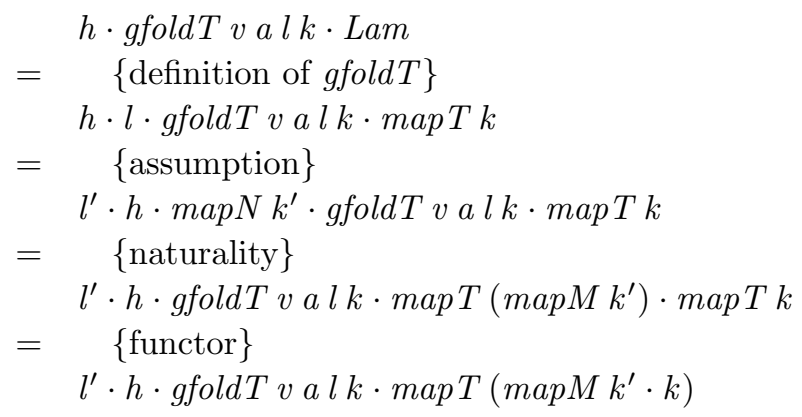

\subsection{A monad}

The type constructor Term is also a monad, with Var as the unit operator, and join $T$ defined by

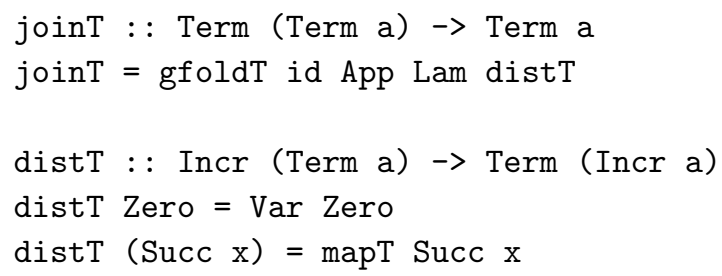


The function distT replaces Succs on terms by Succs on variables. It satisfies the following properties $\dagger$, easily established by cases:

$$
\begin{aligned}
\operatorname{dist} T \cdot \operatorname{mapI} \text { Var } & =\text { Var } \\
\operatorname{dist} T \cdot \operatorname{mapI} \text { join } T & =\operatorname{join} T \cdot \operatorname{map} T \operatorname{dist} T \cdot \operatorname{dist} T
\end{aligned}
$$

Using these equations, and the fusion laws for gfoldT, we can prove the coherence laws for the monad operations on Term:

$$
\begin{aligned}
\text { join } T \cdot \operatorname{Var} & =i d \\
\text { join } T \cdot \operatorname{map} T \text { Var } & =i d \\
\text { join } T \cdot \operatorname{map} T \text { join } T & =\text { join } T \cdot \operatorname{join} T
\end{aligned}
$$

For example, we give the proof of equation (22):

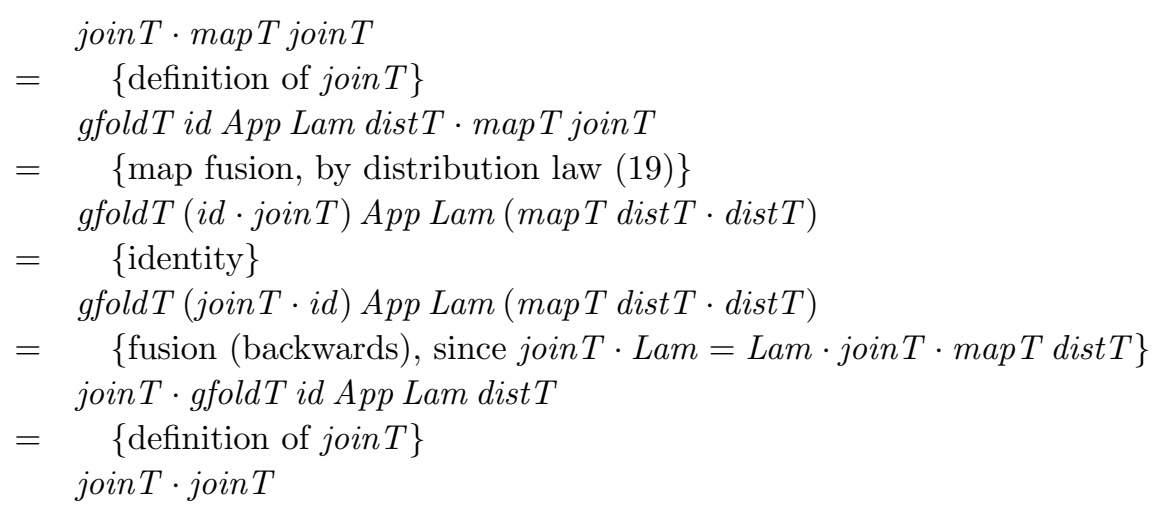

\section{Abstraction and application}

It is time now to return to the main problem in hand, namely, to give the implementations of abstraction and application.

Abstracting with respect to a free variable $x$ is easy: each occurrence of $x$ in a term is replaced by Zero, and each occurrence of a variable $y \neq x$ is replaced by Succ $y$. This is implemented by:

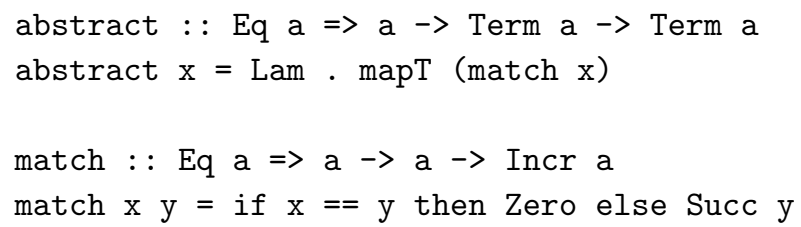

The definition of application is also quite short. We define application as a function that takes a term $t$ and the body $b$ of a lambda abstraction, and replaces every occurrence of Zero (the nameless variable bound by the abstraction) in $b$ by $t$ :

$\dagger$ These equations are part of the statement that dist T is a distributive law (Barr \& Wells, 1984) between the monads on Term and Incr. 


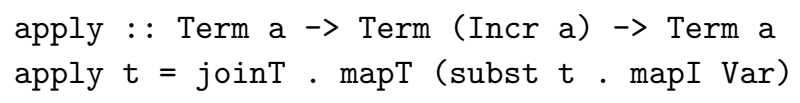

The function mapT (subst $t$ - mapI Var) returns an element of Term (Terma), a term of terms. The function join T 'flattens' such elements into ordinary terms.

The actual substitution is done by the function subst $t$, a left inverse of match $t$ :

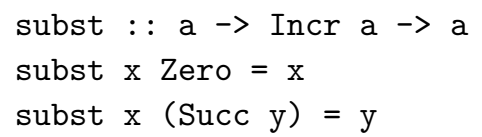

Note that the type of subst implies the following "free theorem" (Wadler, 1989):

$$
f \cdot \text { subst } x=\operatorname{subst}(f x) \cdot \operatorname{mapI} f
$$

To check this definition of apply, let us prove that substituting an abstracted variable returns the original term:

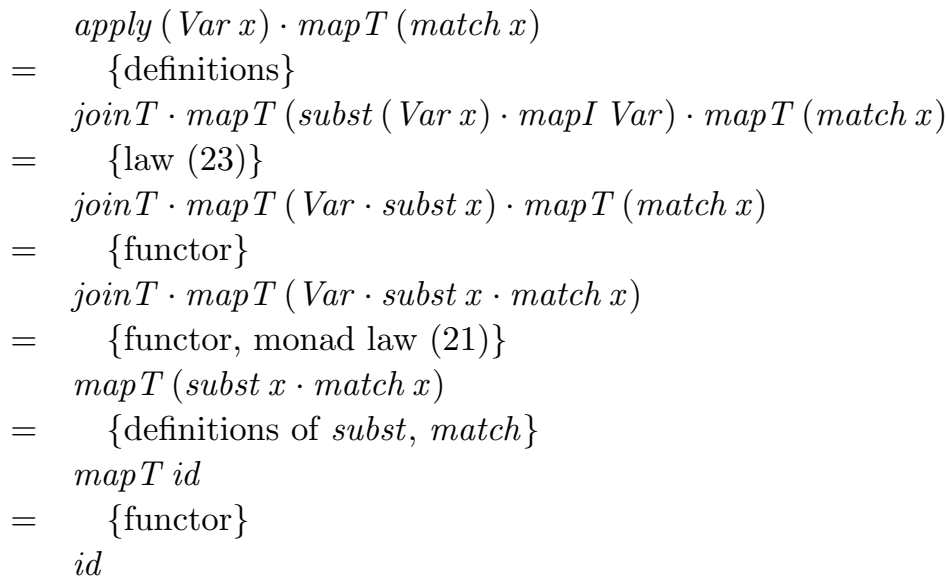

\section{An extension of de Bruijn's notation}

Substitution on de Bruijn terms transforms arguments as well as function bodies, thus precluding sharing. Consider the example term from Section 1, with the variables rewritten in unary notation:

$$
\lambda .0\left(\lambda . S_{0} 0(\lambda . S S 0 \text { S0 0) })\right.
$$

If this term is applied to the term $\lambda .0 \mathrm{~S} 0$, the result is

$$
(\underline{\lambda .0 \mathrm{~S} 0})(\lambda .(\underline{\lambda .0 \mathrm{SS} 0}) 0(\lambda .(\underline{\lambda .0 \mathrm{SSS}})) \mathrm{S} 00))
$$

where the three versions of the argument are underlined. There is a generalisation of de Bruijn notation in which S can be applied to any term, not just a variable (Paterson, 1991). Its effect is to escape the scope of the matching $\lambda$. With this looser representation of terms, one can avoid transforming arguments while substituting. In the above example, substitution yields

$$
(\underline{\lambda .0 \mathrm{~S} 0})\left(\lambda . \mathrm{S}(\underline{\lambda .0 \mathrm{~S} 0}) 0\left(\lambda . \mathrm{SS}(\underline{\lambda .0 \mathrm{~S} 0}) \mathrm{S}_{0} 0\right)\right)
$$


In effect, we have postponed pushing the S's down to the variables.

We still require that each $\mathbf{S}$ or 0 have a matching lambda. This constraint is captured by the following definition:

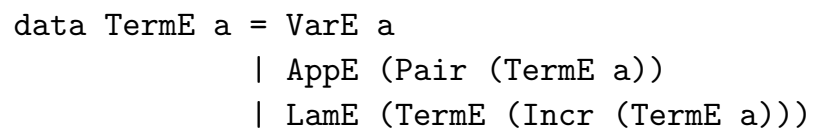

Note that TermE is doubly nested. A similar definition can be used to model quasiquotation (literal data with an escape operator) as in Scheme (Clinger \& Rees, 1991) or multi-stage programming languages like MetaML (Taha \& Sheard, 1997).

Though TermE is more complex, we can follow the same steps as for BinTree and Term. The mapping function for TermE is given by:

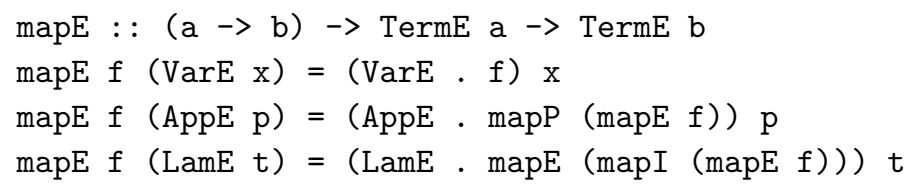

The generalised fold operator is

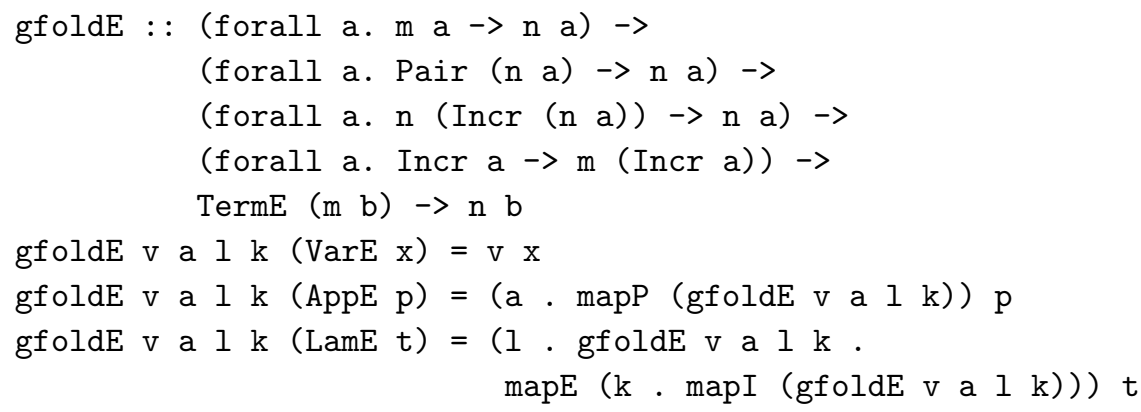

Note the change in type for the last argument $k$ : a lambda abstraction for extended terms with variables of type $m b$ has type

$$
\text { TermE }(\operatorname{Incr}(\operatorname{TermE}(m b)))
$$

Applying mapE ( $m a p I$ (gfoldE v a $l k$ )) to a value of this type produces an element of type

$$
\operatorname{TermE}(\operatorname{Incr}(n b))
$$

Applying mapE $k$ to this element produces an element of type

$$
\operatorname{TermE}(m(\operatorname{Incr}(n b)))
$$

A second recursive application of gfoldE $v$ a $l k$ now produces an element of the type required by $l$, namely,

$$
n(\operatorname{Incr}(n b))
$$

The identity law for extended terms is

$$
\text { gfoldE' VarE AppE LamE id }=i d
$$


The map-fusion law is

$$
\text { gfoldE val }(h \cdot k) \cdot \operatorname{map} E h=\operatorname{gfoldE}(v \cdot h) \text { alk }
$$

The fusion law for gfoldE $v$ a l $k:: \operatorname{TermE}(M a) \rightarrow N a$ is

$$
\begin{aligned}
h \cdot \text { gfoldEvalk } & =\text { gfoldE } v^{\prime} a^{\prime} l^{\prime}\left(\operatorname{map} M k^{\prime} \cdot k\right) \\
& \Leftarrow\left\{\begin{aligned}
h \cdot v=v^{\prime} \\
h \cdot a=a^{\prime} \cdot \operatorname{map} P \\
h \cdot l=l^{\prime} \cdot h \cdot \operatorname{map} N\left(k^{\prime} \cdot \operatorname{map} h\right)
\end{aligned}\right.
\end{aligned}
$$

Extended terms also comprise a monad, with unit $\operatorname{Var} E$ and join operator defined by:

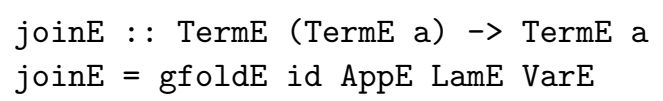

Verification of the monad laws is straightforward. For example, we will prove that

$$
\text { joinE } \cdot \operatorname{map} E \text { joinE }=\text { joinE } \cdot \text { joinE }
$$

We have

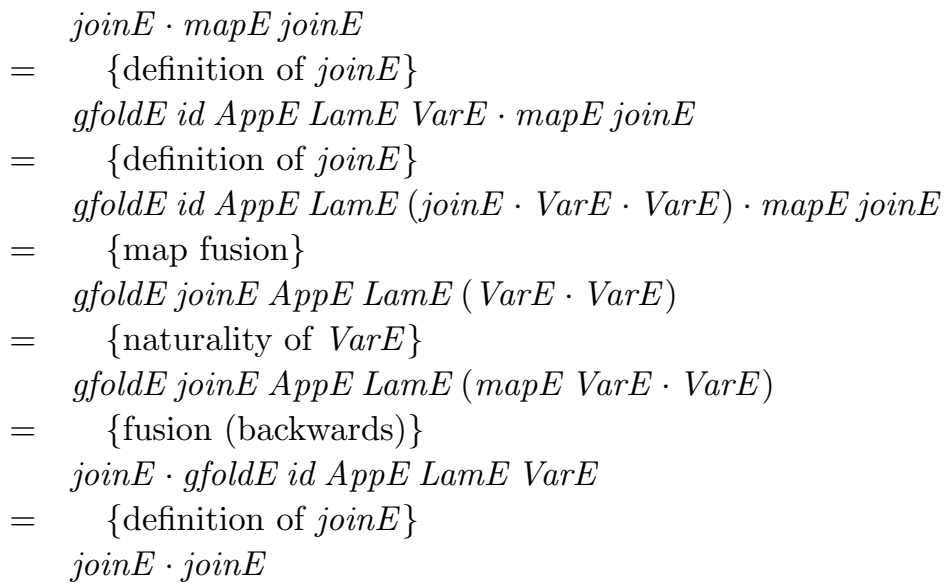

With the definitions above, we can define abstraction and application:

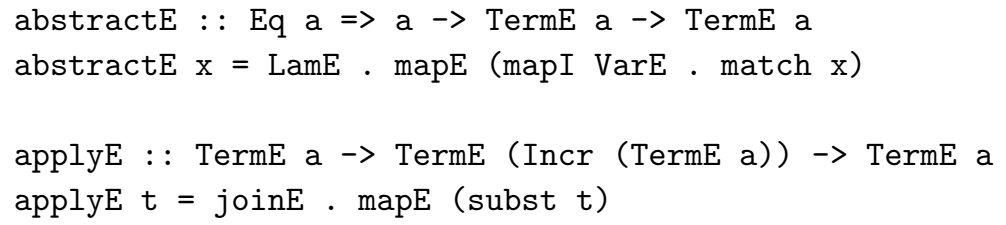

Finally, let us see how to convert extended terms into ordinary ones. We want a function

cvtE : : TermE a $\rightarrow$ Term a

We will define cvtE as an instance of gfoldE. Typing considerations dictate that $m=I d$ and $n=$ Term in the type assignment for gfoldE. Once again Haskell forces us to define a variant $g$ fold $E^{\prime}$, whose definition is the same as that of $g$ foldE, but with $m$ specialised to $I d$. We define 
cvtE = gfoldE' Var App (Lam . joinT . mapT distT) id

To check this definition, we can show that $c v t E$ is a monad morphism, that is, it satisfies the equations:

$$
\begin{aligned}
c v t E \cdot \operatorname{Var} E & =\operatorname{Var} \\
c v t E \cdot j o i n E & =\text { join } T \cdot \operatorname{map} T \operatorname{cvt} E \cdot \operatorname{cvt} E
\end{aligned}
$$

The first is immediate from the definition, and the second is an appeal to fusion:

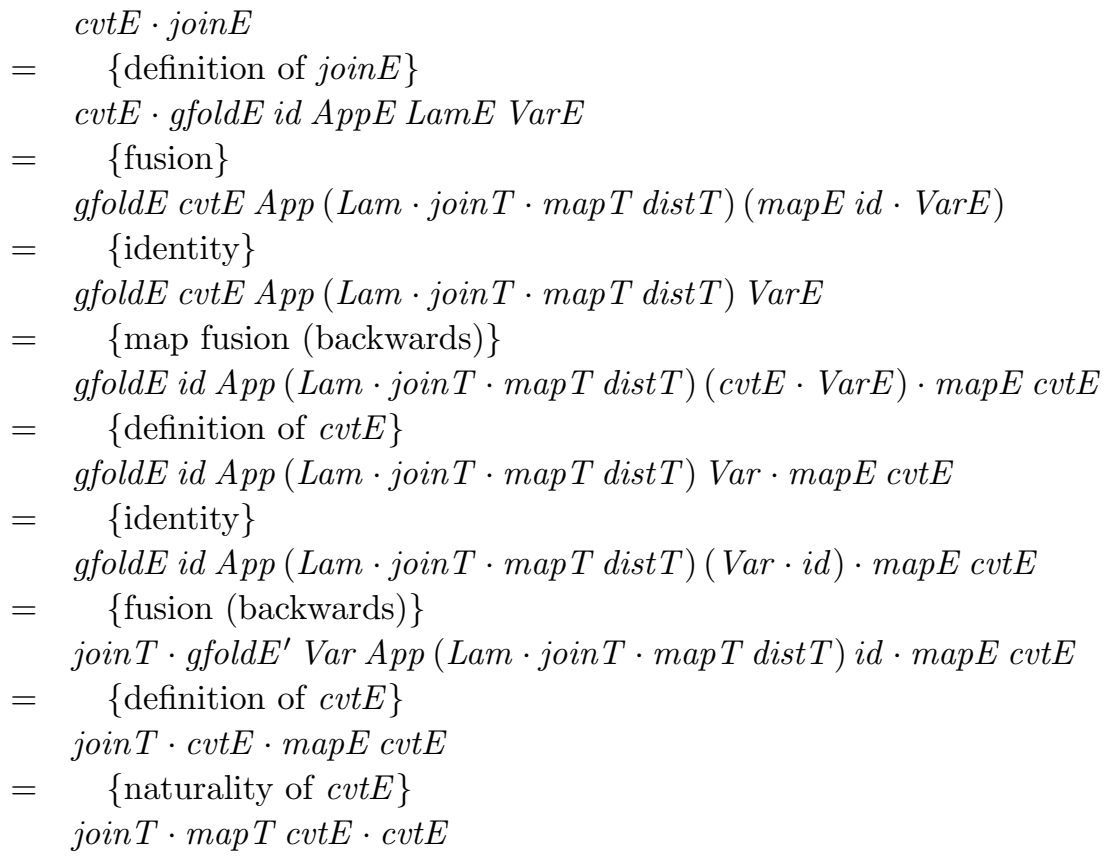

This equation is used in the proof that substitution on extended terms correctly mirrors substitution on de Bruijn terms:

$$
\text { cvtE } \cdot \text { applyE } t=\operatorname{apply}(\operatorname{cvtE} t) \cdot \operatorname{cvtBodyE}
$$

where $\operatorname{cvtBodyE}$ converts an extended abstraction body to a simple one:

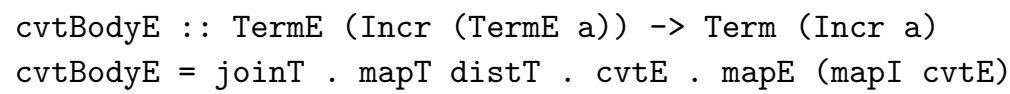

The proof is lengthy but routine, and we omit it.

\section{Conclusion}

Our representation of de Bruijn terms illustrates the ability of nested datatypes to express constraints on data structures, so that they can be enforced by the type checker. It has also served as a test case for the extension to nested datatypes of structuring principles developed for regular datatypes, using maps, folds and monads. In the case of de Bruijn terms, these operators do most of the work, including 
handling bound variables, so that the definition of application and abstraction is particularly simple. Moreover, with programs structured in this way most proofs are mechanical, and were indeed generated using the simple automatic calculator described in (Bird, 1998).

Programs that manipulate nested types require a number of recently explored extensions of the Hindley-Milner type system. The limited form of type constructor polymorphism provided by Haskell has been an occasional hindrance, forcing us to define specialised versions of polymorphic functions, or new datatypes that are equivalent to existing types; in both cases an opportunity for reuse is lost. It might be reasonable to design a language in which these restrictions were lifted, at the cost of explicit abstraction and instantiation with respect to type constructors, but not types.

\section{Acknowledgements}

Oege de Moor suggested using a nested datatype for lambda terms. An anonymous referee suggested a number of improvements.

\section{References}

Barr, Michael, \& Wells, Charles. (1984). Toposes, triples and theories. Grundlehren der Mathematischen Wissenschaften, no. 278. New York: Springer.

Bird, R., \& de Moor, O. (1997). The algebra of programming. Prentice Hall.

Bird, Richard. (1998). Introduction to functional programming using Haskell. Second edn. Prentice Hall.

Bird, Richard, \& Paterson, Ross. (1998). Generalised folds for nested datatypes. in preparation.

Bird, Richard S., \& Meertens, Lambert. (1998). Nested datatypes. Pages 52-67 of: Mathematics of program construction. Lecture Notes in Computer Science, vol. 1422. Springer.

Clinger, W., \& Rees, J. (1991). Revised ${ }^{4}$ report on the algorithmic language Scheme. ACM Lisp pointers, IV(July-Sept.).

de Bruijn, N.G. (1972). Lambda calculus notation with nameless dummies. Indagationes mathematicae, 34, 381-392.

Jones, Mark. (1998). A technical summary of the new features in Hugs 1.3c. unpublished.

McCracken, N. J. (1984). The typechecking of programs with implicit type structure. Pages 301-315 of: Semantics of data types. Lecture Notes in Computer Science, vol. 173. Springer.

Paterson, R.A. (1991). Non-deterministic lambda-calculus: A core for integrated languages. Declarative programming, Sassbachwalden. Springer.

Paulson, L.C. (1996). ML for the working programmer. Second edn. Cambridge University Press.

Peyton Jones, Simon, et al. . (1998). The Glasgow Haskell compiler. Department of Computer Science, University of Glasgow.

Taha, Walid, \& Sheard, Tim. (1997). Multi-stage programming with explicit annotations. Pages 203-217 of: ACM symposium of partial evaluation and semantics-based program manipulation.

Wadler, Philip. (1989). Theorems for free! Pages 347-359 of: 4th conference on functional programming languages and computer architecture. IFIP. 